\title{
An Approximate Analytical Solution of the Nonlinear Schrödinger Equation with Harmonic Oscillator Using Homotopy Perturbation Method and Laplace-Adomian Decomposition Method
}

\author{
Emad K. Jaradat, ${ }^{1}$ Omar Alomari $\left(\mathbb{D},{ }^{2}\right.$ Mohammad Abudayah ${ }^{D},{ }^{2}$ and Ala'a M. Al-Faqih ${ }^{1}$ \\ ${ }^{1}$ Department of physics, Mutah University, Jordan \\ ${ }^{2}$ German Jordanian University, Amman, Jordan
}

Correspondence should be addressed to Omar Alomari; omar.alomari@gju.edu.jo

Received 2 October 2018; Revised 22 November 2018; Accepted 26 November 2018; Published 5 December 2018

Academic Editor: Antonio Scarfone

Copyright (C) 2018 Emad K. Jaradat et al. This is an open access article distributed under the Creative Commons Attribution License, which permits unrestricted use, distribution, and reproduction in any medium, provided the original work is properly cited.

The Laplace-Adomian Decomposition Method (LADM) and Homotopy Perturbation Method (HPM) are both utilized in this research in order to obtain an approximate analytical solution to the nonlinear Schrödinger equation with harmonic oscillator. Accordingly, nonlinear Schrödinger equation in both one and two dimensions is provided to illustrate the effects of harmonic oscillator on the behavior of the wave function. The available literature does not provide an exact solution to the problem presented in this paper. Nevertheless, approximate analytical solutions are provided in this paper using LADM and HPM methods, in addition to comparing and analyzing both solutions.

\section{Introduction}

The Schrödinger equation is often encountered in many branches of science and engineering, including quantum mechanics, nonlinear optics, plasma physics, hydrodynamics, and superconductivity. It is a mathematical partial differential equation used to describe the motion and behavior change of the physical system over time. In classical mechanics, it plays the role of Newton's law and conservation of energy. In quantum mechanics, we describe systems using wave function. The Schrödinger equation has two "forms"; one is the time-dependent wave equation that describes how the wave function of a particle will evolve in time. The other is the time independent wave equation in which the time dependence has been "removed"; it describes what the allowed energies are of the particle $[1,2]$.

In recent years, a considerable amount of research focused on finding analytical solution to the Schrödinger equations using various methods, among which are Adomian Decomposition Method [3-8], Elzaki decomposition method [9], Variation Iteration method [10], Nikiforod-Uvarov (NV) method [11], and Homotopy Perturbation Method [3, 4, 12-16]. Additionally, Borhanifar [17] solved the nonlinear Schrödinger and coupled Schrödinger equations with a differential transformation method. Shidfar and Molabahrami $[18,19]$ investigated the d-dimensional Schrödinger equation with a power-law nonlinearity, Zhenga et al. [20] solved the time-dependent Schrödinger equation using homotopy analysis method (HAM) and the Adomian decomposition technique (ADM), and Amador et al. [21] solved nonlinear Schrödinger equations with variable coefficients using Riccati equations and similarity transformations. Finally, Khan and $\mathrm{Wu}$ [22] applied Homotopy perturbation transform method (HPTM) to solve nonlinear equations; HPTM uses the Homotopy Perturbation Method together with the Laplace transformation to solve the nonlinear equations. Also, Hosseini et al. [23-27] investigated various forms of the nonlinear Schrödinger equation (NLSE).

This paper is organized in several sections. The HPM method is briefly explained in "Homotopy Perturbation Method". Then the LADM model is described in the "Laplace-Adomian Decomposition Method". Then in the 
"One-Dimensional Nonlinear Schrödinger Equation with Harmonic Oscillator", the solution to the One-Dimensional Nonlinear Schrödinger Equation with Harmonic Oscillator equation in its nonlinear version is provided with a numerical example. Similarly, the solution of the Two-Dimensional Nonlinear Schrödinger Equation with Harmonic Oscillator is presented in the "Two-Dimensional Nonlinear Schrödinger Equation with Harmonic Oscillator". Finally, in the "Conclusion", we summarize our findings and present our final remarks. Since the exact solution to this problem is not available, we compare our numerical results with the results obtained using Mathematica function NDsolve.

\section{Laplace-Adomian Decomposition Method}

The Adomian Decomposition Method (ADM) is a method to solve differential equations by expressing the analytic solution in terms of a series. The method separates the linear and nonlinear parts of a differential equation. The nonlinear part can be expressed in terms of what is called Adomian Polynomials [28-30]. The initial condition and the terms that contain the independent variables will be used as the initial approximation. Then by means of a recurrence relation, it is possible to find the terms of the series that give the approximate solution of the differential equation.

The Laplace transform is an integral transform that is powerful and useful technique to solve differential equations, which transforms the original differential equation into an algebraic equation.

Below are the definitions of Laplace transform and inverse Laplace transform.

Definition 1. Given a function $f(t)$ defined for all $t \geq 0$, the Laplace transform of $f$ is the function $F$ defined by

$$
F(s)=\mathscr{L}\{f(t)\}=\int_{0}^{\infty} f(t) e^{-s t} d t,
$$

and the inverse Laplace transform is defined as follows.

Definition 2. Given a continuous function $f(t)$, if $F(s)=$ $\mathscr{L}\{f(t)\}$, then $f(t)$ is called the inverse Laplace transform of $F(s)$ denoted $f(t)=\mathscr{L}^{-1}\{F(s)\}$.

The Laplace-Adomian Decomposition Method (LADM) was first introduced by Suheil A. Khuri [31] and has been effectively used to find solutions to general nonlinear equations. The added value of this method utilizes the two methods (Laplace Transform and ADM) to obtain the solution for nonlinear equations. Consider the following equation:

$$
\begin{aligned}
L \Psi-i R \Psi-i N \Psi & =0, \\
\Psi(x, 0) & =f(x),
\end{aligned}
$$

where $L=\partial / \partial t$ and $R=\partial^{2} / \partial x^{2}, L$ and $R$ are Linear operators, and $N$ is a nonlinear operator.

Laplace-Adomian Decomposition Method consists of applying Laplace transform to both sides of (2) and yields

$$
\mathscr{L}\{L \Psi\}-\mathscr{L}\{i R \Psi\}-\mathscr{L}\{i N \Psi\}=0 .
$$

From Laplace transform of first derivative and substituting the initial condition, we get

$$
\mathscr{L}\{\Psi(x, t)\}=\frac{f(x)}{s}+\frac{i}{s} \mathscr{L}\{R \Psi+N \Psi\} .
$$

Next step is replacing the wave function by an infinite series of terms to be determined later as per the Adomian Decomposition Method (ADM):

$$
\Psi(x, t)=\sum_{n=0}^{\infty} \Psi_{n}(x, t),
$$

and the nonlinear terms are replaced by the series:

$$
N \Psi=\sum_{n=0}^{\infty} A_{n}\left(\Psi_{0}, \Psi_{1}, \ldots, \Psi_{n}\right),
$$

where $A_{n}\left(\Psi_{0}, \Psi_{1}, \ldots\right)$ 's are the Adomian Polynomials, defined by

$$
A_{n}=\left.\frac{1}{n !}\left[\frac{d^{n}}{d \lambda^{n}} N\left(\sum_{n=0}^{\infty} \lambda^{i} \Psi_{i}\right)\right]\right|_{\lambda=0}, \quad n=0,1,2 \ldots
$$

Substituting (5) and (6) into (4) and taking inverse Laplace transform, we get

$$
\begin{aligned}
\sum_{n=0}^{\infty} \Psi_{n}(x, t) & \\
= & f(x) \\
& +\mathscr{L}^{-1}\left\{\frac{i}{s}\left(\mathscr{L}\left\{R \sum_{n=0}^{\infty} \Psi_{n}(x, t)+\sum_{n=0}^{\infty} A_{n}\right\}\right)\right\} .
\end{aligned}
$$

From (8), one can obtain

$$
\begin{gathered}
\Psi_{0}(x, t)=\Psi(x, 0)=f(x), \\
\Psi_{1}(x, t)=i \mathscr{L}^{-1}\left\{\frac{1}{s} \mathscr{L}\left\{R \Psi_{0}(x, t)+A_{0}\right\}\right\}, \\
\Psi_{2}(x, t)=i \mathscr{L}^{-1}\left\{\frac{1}{s} \mathscr{L}\left\{R \Psi_{1}(x, t)+A_{1}\right\}\right\}, \\
\vdots \\
\Psi_{n}(x, t)=i \mathscr{L}^{-1}\left\{\frac{1}{s} \mathscr{L}\left\{R \Psi_{n-1}(x, t)+A_{n-1}\right\}\right\} .
\end{gathered}
$$

\section{Homotopy Perturbation Method}

The Homotopy Perturbation Method (HPM) is a special case of the homotopy analysis method (HAM) [32]. HPM was presented by He [33] in 1999, and is considered as a strong and efficient technique in finding an exact or an approximate analytical solutions to nonlinear equations.

To demonstrate the idea of Homotopy Perturbation Method, we consider the general form nonlinear differential equation with initial conditions of the form [3]:

$$
\begin{aligned}
L(\Psi)+N(\Psi)-f(r) & =0, \quad r \in \Omega, \\
B\left(\Psi, \frac{\partial \Psi}{\partial n}\right) & =0, \quad r \in \Gamma,
\end{aligned}
$$


where $L$ and $N$ are linear and nonlinear operators respectively, $f(r)$ is an analytic function, $\Gamma$ is the boundary of the domain $\Omega$, and $\partial \Psi / \partial n$ denotes the differentiation of $\Psi$ with respect to $n$.

To apply the Homotopy concept to (10), we construct a suitable Homotopy,

$$
v(r, p): \Omega \times[0,1] \longrightarrow \mathbb{R},
$$

that satisfies

$$
\begin{aligned}
H(v, p)= & (1-p)\left[L(v)-L\left(\Psi_{0}\right)\right] \\
& +p[L(v)+N(v)-f(r)]=0, \quad r \in \Omega,
\end{aligned}
$$

where $p \in[0,1]$ is a parameter which increases from 0 to $1, \mathbb{R}$ represents all real numbers, and $\Psi_{0}$ is an initial approximate solution of (10), which satisfies the boundary conditions (11). Clearly, from (13) we have

$$
\begin{aligned}
& H(v, 0)=L(v)-L\left(\Psi_{0}\right)=0, \\
& H(v, 1)=L(v)+N(v)-f(r)=0 .
\end{aligned}
$$

Now, when the value of $p$ changes from 0 to $1, v(r, p)$ changes from $\Psi_{0}$ to $\Psi(r)$. According to the concept of topology, this is called deformation and $L(v)-N\left(\Psi_{0}\right)$ and $L(v)-f(r)$ are called homotopy. If we consider $p$ as a small parameter, then applying the original perturbation technique method, we can assume that the solution of (13) can be defined as a power series in $p$ :

$$
v=v_{0}+v_{1} p+v_{2} p^{2}+\cdots
$$

Then the solution $\Psi$ to (10) is obtained as $p$ approaches 1:

$$
\Psi=\lim _{p \longrightarrow 1} v=v_{0}+v_{1}+v_{2}+\cdots
$$

The series in (17) is convergent for most cases, the rate of convergence however is dependent on the nonlinear operator $N(v)[33]$.

In the following two sections we apply the above two methods to solve the One- and Two-Dimensional Nonlinear Schrödinger Equation with Harmonic Oscillator.

\section{One-Dimensional Nonlinear Schrödinger Equation with Harmonic Oscillator}

The nonlinear Schrödinger equation with harmonic oscillator described by $\Psi$ with identical initial condition can be expressed as [20]

$$
\begin{array}{r}
\frac{\partial \Psi}{\partial t}-\frac{i}{2 m} \frac{\partial^{2} \Psi}{\partial x^{2}}+\frac{i}{2} k x^{2} \Psi+i|\Psi|^{2} \Psi=0, \\
\Psi(x, 0)=e^{i x},
\end{array}
$$

where $\Psi$ is the wave function, $m$ is the mass of the particle, $i$ is the imaginary unit to describe motion, and $k$ spring constant.
4.1. $L A D M$. In this section we solve the One-Dimensional Nonlinear Schrödinger Equation with Harmonic Oscillator (18) using LADM method by first applying Laplace transform to both sides of the equation (18) as follows:

$$
\begin{gathered}
\mathscr{L}\left\{\frac{\partial \Psi}{\partial t}\right\}-i \mathscr{L}\left\{\frac{1}{2 m} \frac{\partial^{2} \Psi}{\partial x^{2}}\right\}+i \mathscr{L}\left\{\frac{1}{2} k x^{2} \Psi\right\} \\
+i \mathscr{L}\left\{|\Psi|^{2} \Psi\right\}=0 .
\end{gathered}
$$

From the properties of Laplace transform of the first derivative and substituting the initial conditions (19), (20) becomes

$$
\begin{aligned}
\mathscr{L}\{\Psi(x, t)\}= & \frac{e^{i x}}{s}+\frac{1}{s} \mathscr{L}\left\{\frac{i}{2 m} \frac{\partial^{2} \Psi}{\partial x^{2}}\right\}-\frac{1}{s} \frac{i k}{2} \mathscr{L}\left\{x^{2} \Psi\right\} \\
& -\frac{1}{s} \mathscr{L}\left\{i|\Psi|^{2} \Psi\right\} .
\end{aligned}
$$

Now substituting (5) and (6) into (21), we get

$$
\begin{aligned}
\mathscr{L}\left\{\sum_{n=0}^{\infty} \Psi_{n}(x, t)\right\} \\
=\frac{e^{i x}}{s}+\frac{1}{s} \mathscr{L}\left\{\frac{i}{2 m} \frac{\partial^{2}}{\partial x^{2}}\left(\sum_{n=0}^{\infty} \Psi_{n}(x, t)\right)\right\} \\
\quad-\frac{i k}{2 s} \mathscr{L}\left\{x^{2} \sum_{n=0}^{\infty} \Psi_{n}(x, t)\right\}-\frac{i}{s} \mathscr{L}\left\{\sum_{n=0}^{\infty} A_{n}\right\} .
\end{aligned}
$$

By applying inverse Laplace transform to (22) and taking into consideration

$$
\mathscr{L}^{-1}\left\{\mathscr{L}\left\{\Psi_{0}(x, t)\right\}\right\}=\mathscr{L}^{-1}\left\{\frac{e^{i x}}{s}\right\},
$$

we have

$$
\begin{aligned}
\Psi_{n}(x, t)= & \mathscr{L}^{-1}\left\{\frac{i}{2 m s} \mathscr{L}\left\{\frac{\partial^{2} \Psi_{n-1}(x, t)}{\partial x^{2}}\right\}\right\} \\
& -\mathscr{L}^{-1}\left\{\frac{i k}{2 s} \mathscr{L}\left\{x^{2} \Psi_{n-1}(x, t)\right\}\right\} \\
& -\mathscr{L}^{-1}\left\{\frac{i}{s} \mathscr{L}\left\{A_{n-1}\right\}\right\},
\end{aligned}
$$

where $A_{n}$ are the so called Adomian Polynomials of $\left(\Psi_{0}, \Psi_{1}, \Psi_{2}, \ldots, \Psi_{n}\right)$ to replace $N \Psi=|\Psi|^{2} \Psi=\Psi^{2} \bar{\Psi}$ and $\bar{\Psi}$ is the conjugate of $\Psi$.

The Adomian Polynomials can be calculated using (7):

$$
\begin{aligned}
& A_{0}=\Psi_{0}^{2} \bar{\Psi}_{0} \\
& A_{1}=2 \Psi_{0} \Psi_{1} \bar{\Psi}_{0}+\Psi_{0}^{2} \bar{\Psi}_{1} \\
& A_{2}=2 \Psi_{0} \Psi_{2} \bar{\Psi}_{0}+\Psi_{1}^{2} \bar{\Psi}_{0}+2 \Psi_{0} \Psi_{1} \bar{\Psi}_{1}+\Psi_{0}^{2} \bar{\Psi}_{2}
\end{aligned}
$$


Now, using (24), we get

$$
\begin{aligned}
& \Psi_{0}(x, t)=e^{i x}, \\
& \Psi_{1}(x, t)=-\frac{i t e^{i x}}{2 m}-\frac{i k x^{2} t e^{i x}}{2}-i t e^{i x}, \\
& \Psi_{2}(x, t)=\frac{t^{2}}{2 !}\left(\frac{-e^{i x}}{4 m^{2}}+\frac{k e^{i x}}{2 m}+\frac{i k x e^{i x}}{m}-\frac{e^{i x}}{m}-\frac{k x^{2} e^{i x}}{2 m}\right. \\
& \left.-\frac{k^{2} x^{4} e^{i x}}{4}-k x^{2} e^{i x}-e^{i x}\right), \\
& \Psi_{3}(x, t)=\frac{t^{3}}{3 !}\left(\frac{3 i e^{i x}}{4 m^{2}}+\frac{3 i k x^{2} e^{i x}}{8 m^{2}}+\frac{i e^{i x}}{8 m^{3}}+\frac{3 k x e^{i x}}{2 m^{2}}\right. \\
& -\frac{7 i k e^{i x}}{4 m^{2}}-\frac{3 i k e^{i x}}{2 m}+\frac{3 i k x^{2} e^{i x}}{2 m}+\frac{3 i e^{i x}}{2 m}+\frac{i k^{3} x^{6} e^{i x}}{8} \\
& +\frac{i 3 k^{2} x^{4} e^{i x}}{4}+i e^{i x}+\frac{3 k x e^{i x}}{m}+\frac{3 i k^{2} x^{4} e^{i x}}{8 m} \\
& \left.-\frac{7 i k^{2} x^{2} e^{i x}}{4 m}+\frac{3 k^{2} x^{3} e^{i x}}{2 m}+\frac{3 i k x^{2} e^{i x}}{2}\right)
\end{aligned}
$$

Therefore, the solution $\Psi(x, t)$ is given by

$$
\begin{aligned}
& \Psi(x, t)=\Psi_{0}+\Psi_{1}+\Psi_{2}+\cdots \Psi_{n}=e^{i x}\left[1+\left(-\frac{i t}{2 m}\right.\right. \\
& \left.-\frac{i k x^{2} t}{2}-i t\right)+\frac{1}{2 !}\left(\frac{-i t}{2 m}-\frac{i k x^{2} t}{2}-i t\right)^{2} \\
& +\frac{t^{2}}{2 !}\left(\frac{k}{2 m}+\frac{i k x}{m}\right)+\frac{1}{3 !}\left(\frac{-i t}{2 m}-\frac{i k x^{2} t}{2}-i t\right)^{3} \\
& +\frac{t^{3}}{3 !}\left(\frac{3 k x}{2 m^{2}}-\frac{7 i k}{4 m^{2}}-\frac{3 i k}{2 m}+\frac{3 k x}{m}-\frac{7 i k^{2} x^{2}}{4 m}\right. \\
& \left.\left.+\frac{3 k^{2} x^{3}}{2 m}\right)+\cdots\right]
\end{aligned}
$$

4.2. HPM. In this section we apply the Homotopy Perturbation Method to obtain a solution to (18). Consider the following homotopy:

$$
\begin{aligned}
H(v, p)= & (1-p)\left(\frac{\partial v}{\partial t}-\frac{\partial \Psi_{0}}{\partial t}\right) \\
& +p\left(\frac{\partial v}{\partial t}-\frac{i \partial^{2} v}{2 m \partial x^{2}}+\frac{i}{2} k x^{2} v+i v^{2} \bar{v}\right)=0
\end{aligned}
$$

where $\Psi_{0}=\Psi(x, 0), \partial \Psi_{0} / \partial t=\partial \Psi(x, 0) / \partial t$, and $\bar{v}(x, t)$ is the complex conjugate of $v(x, t)$. Suppose that the series solution $v$ of (28) and its conjugate $\bar{v}$ have the following forms:

$$
v(x, t)=v_{0}(x, t)+v_{1}(x, t) p+v_{2}(x, t) p^{2}+\cdots,
$$
and

$$
\bar{v}(x, t)=\bar{v}_{0}(x, t)+\bar{v}_{1}(x, t) p+\bar{v}_{2}(x, t) p^{2}+\cdots,
$$

and hence, the solution to (18) is

$$
\Psi(x, t)=v_{0}(x, t)+v_{1}(x, t)+v_{2}(x, t)+\cdots .
$$

Substituting (29) and (30) into (28) and equating the coefficients of $p$ powers, we have

$$
\begin{aligned}
& p^{0}: \frac{\partial v_{0}}{\partial t}-\frac{\partial \Psi_{0}}{\partial t}=0, \\
& \Psi_{0}(x, 0)=e^{i x} \\
& p^{1}: \frac{\partial v_{1}}{\partial t}=\frac{i \partial^{2} v_{0}}{2 m \partial x^{2}}-\frac{i}{2} k x^{2} v_{0}-i\left|v_{0}\right|^{2} v_{0}, \\
& v_{1}(x, 0)=0, \\
& p^{2}: \frac{\partial v_{2}}{\partial t}=\frac{i \partial^{2} v_{1}}{2 m \partial x^{2}}-\frac{i}{2} k x^{2} v_{1}-i\left[v_{0}^{2} \overline{v_{1}}+2 v_{0} \overline{v_{0}} v_{1}\right], \\
& v_{2}(x, 0)=0,
\end{aligned}
$$

$$
\begin{aligned}
& p^{j}: \frac{\partial v_{j}}{\partial t}=\frac{i \partial^{2} v_{j-1}}{2 m \partial x^{2}}-\frac{i}{2} k x^{2} v_{j-1} \\
& \quad-i\left(\sum_{i=0}^{i-1} \sum_{k=0}^{j-i-1}\left|v_{i}\right|\left|v_{k}\right| \mid v_{j-k-i-1}\right), \\
& v_{j}(x, 0)=0 .
\end{aligned}
$$

We finally obtain the general solution of (18) given the recurrence relation for $j=1,2,3, \ldots$

$$
\begin{aligned}
v_{j} & =\int_{0}^{t}\left(\frac{i \partial^{2} v_{j-1}}{2 m \partial x^{2}}-\frac{i}{2} k x^{2} v_{j-1}\right. \\
& \left.-i\left(\sum_{i=0}^{i-1} \sum_{k=0}^{j-i-1}\left|v_{i}\right|\left|v_{k}\right| \mid v_{j-k-i-1}\right)\right) d \tau .
\end{aligned}
$$

The first few terms of the solution are calculated as follows:

$$
\begin{aligned}
& v_{0}(x, t)=e^{i x}, \\
& v_{1}(x, t)=-\frac{i t e^{i x}}{2 m}-\frac{i k x^{2} t e^{i x}}{2}-i t e^{i x}, \\
& v_{2}(x, t)=\frac{t^{2}}{2 !}\left(\frac{-e^{i x}}{4 m^{2}}+\frac{k e^{i x}}{2 m}+\frac{i k x e^{i x}}{m}-\frac{e^{i x}}{m}-\frac{k x^{2} e^{i x}}{2 m}\right. \\
& \left.-\frac{k^{2} x^{4} e^{i x}}{4}-k x^{2} e^{i x}-e^{i x}\right),
\end{aligned}
$$




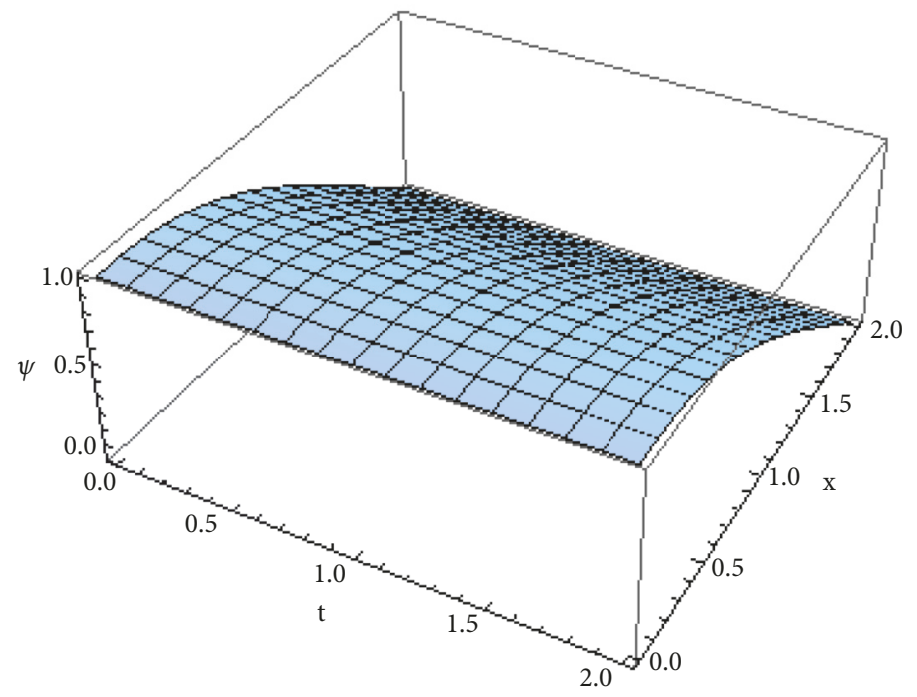

FIGURE 1: Real solution of the one-dimensional wave function obtained by LADM and HPM.

$$
\begin{aligned}
& v_{3}(x, t)=\frac{t^{3}}{3 !}\left(\frac{3 i e^{i x}}{4 m^{2}}+\frac{3 i k x^{2} e^{i x}}{8 m^{2}}+\frac{i e^{i x}}{8 m^{3}}+\frac{3 k x e^{i x}}{2 m^{2}}\right. \\
& -\frac{7 i k e^{i x}}{4 m^{2}}-\frac{3 i k e^{i x}}{2 m}+\frac{3 i k x^{2} e^{i x}}{2 m}+\frac{3 i e^{i x}}{2 m}+\frac{i k^{3} x^{6} e^{i x}}{8} \\
& +\frac{i 3 k^{2} x^{4} e^{i x}}{4}+i e^{i x}+\frac{3 k x e^{i x}}{m}+\frac{3 i k^{2} x^{4} e^{i x}}{8 m} \\
& \left.-\frac{7 i k^{2} x^{2} e^{i x}}{4 m}+\frac{3 k^{2} x^{3} e^{i x}}{2 m}+\frac{3 i k x^{2} e^{i x}}{2}\right),
\end{aligned}
$$

and hence, the solution to (18) is

$$
\begin{aligned}
& \Psi(x, t)=v_{0}(x, t)+v_{1}(x, t)+v_{2}(x, t)+v_{3}(x, t) \\
& +\cdots=e^{i x}\left[1+\left(-\frac{i t}{2 m}-\frac{i k x^{2} t}{2}-i t\right)+\frac{1}{2 !}\left(\frac{-i t}{2 m}\right.\right. \\
& \left.-\frac{i k x^{2} t}{2}-i t\right)^{2}+\frac{t^{2}}{2 !}\left(\frac{k}{2 m}+\frac{i k x}{m}\right)+\frac{1}{3 !}\left(\frac{-i t}{2 m}\right. \\
& \left.-\frac{i k x^{2} t}{2}-i t\right)^{3}+\frac{t^{3}}{3 !}\left(\frac{3 k x}{2 m^{2}}-\frac{7 i k}{4 m^{2}}-\frac{3 i k}{2 m}+\frac{3 k x}{m}\right. \\
& \left.\left.-\frac{7 i k^{2} x^{2}}{4 m}+\frac{3 k^{2} x^{3}}{2 m}\right)+\cdots\right] .
\end{aligned}
$$

The graphs of the one-dimensional wave function are shown in Figures 1, 2, 3, and 4. Figure 1 is the graph of the third order approximation for the solution of the real part of the wave function obtained by LADM and HPM. Figure 2 is the graph of the wave function obtained using Mathematica function NDsolve. Figures 3 and 4 show the imaginary part of the solution. We use $k=m=1$ in the calculations.

\section{Two-Dimensional Nonlinear Schrödinger Equation with Harmonic Oscillator}

In this section we look at a particle movement in two dimensions, the nonlinear Schrödinger equation with harmonic oscillator when a particle moves in two dimensions with the initial condition can be written as [20]

$$
\begin{aligned}
& \frac{\partial \Psi}{\partial t}-\frac{i}{2 m}\left(\frac{\partial^{2} \Psi}{\partial x^{2}}+\frac{\partial^{2} \Psi}{\partial y^{2}}\right)+\frac{i k}{2}\left(x^{2}+y^{2}\right) \Psi \\
& +i|\Psi|^{2} \Psi=0, \\
& \Psi(x, y, 0)=e^{i(x+y)} .
\end{aligned}
$$

5.1. LADM. Similar to the one-dimensional, after applying Laplace transform to (36), substituting the initial condition, and making $\mathscr{L}\{\Psi(x, y, t)\}$ the subject, we have

$$
\begin{aligned}
\mathscr{L}\{\Psi(x, y, t)\}= & \frac{e^{i(x+y)}}{s} \\
& +\frac{1}{s} \mathscr{L}\left\{\frac{i}{2 m}\left(\frac{\partial^{2} \Psi}{\partial x^{2}}+\frac{\partial^{2} \Psi}{\partial y^{2}}\right)\right\} \\
& -\frac{1}{s} \mathscr{L}\left\{\frac{i k}{2}\left(x^{2}+y^{2}\right) \Psi\right\} \\
& -\frac{1}{s} \mathscr{L}\left\{i|\Psi|^{2} \Psi\right\} .
\end{aligned}
$$

Replacing the wave function $\Psi(x, y)$ and $|\Psi|^{2} \Psi$ in (38) by the infinite series below,respectively,

$$
\Psi(x, y, t)=\sum_{n=0}^{\infty} \Psi_{n}(x, y, t)
$$




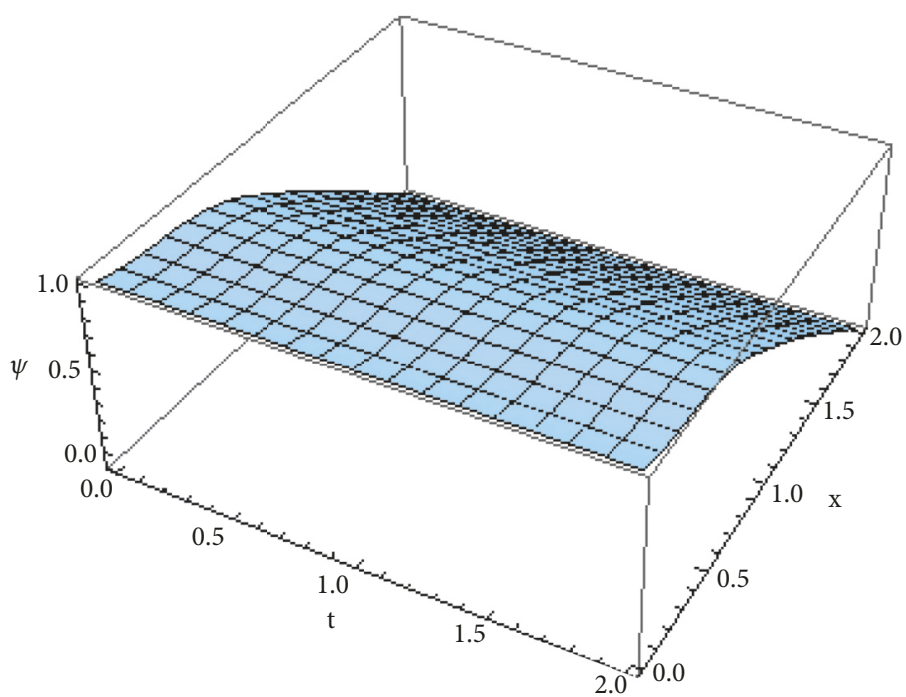

FIGURE 2: Real solution of the one-dimensional wave function obtained by NDsolve.

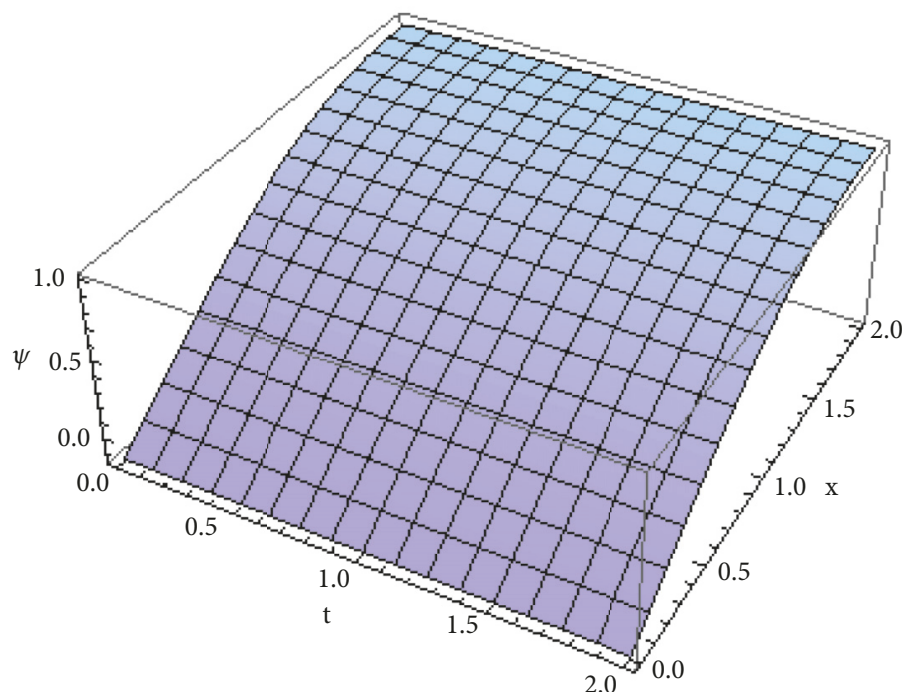

FIGURE 3: Imaginary solution of the one-dimensional wave function obtained by LADM and HPM.

and

$$
|\Psi|^{2} \Psi=\sum_{n=0}^{\infty} A_{n}\left(\Psi_{0}, \Psi_{1}, \ldots, \Psi_{n}\right),
$$

$$
\begin{aligned}
& \text { we get } \\
& \qquad \mathscr{L}\left\{\sum_{n=0}^{\infty} \Psi_{n}(x, y, t)\right\}=\frac{e^{i(x+y)}}{s}+\frac{1}{s} \\
& . \mathscr{L}\left\{\frac { i } { 2 m } \left(\frac{\partial^{2}}{\partial x^{2}}\left(\sum_{n=0}^{\infty} \Psi_{n}(x, y, t)\right)\right.\right. \\
& \left.\left.+\frac{\partial^{2}}{\partial y^{2}}\left(\sum_{n=0}^{\infty} \Psi_{n}(x, y, t)\right)\right)\right\}-\frac{1}{s} \mathscr{L}\left\{\frac{i k}{2}\left(x^{2}+y^{2}\right)\right. \\
& \left.. \sum_{n=0}^{\infty} \Psi_{n}(x, y, t)\right\}-\frac{1}{s} \mathscr{L}\left\{i\left(\sum_{n=0}^{\infty} A_{n}\right)\right\} .
\end{aligned}
$$

Applying inverse Laplace transform, we have

$$
\begin{aligned}
\Psi_{0}= & \mathscr{L}^{-1}\left\{\frac{e^{i(x+y)}}{s}\right\}, \\
\Psi_{1} & \mathscr{L}^{-1}\left\{\frac{i}{2 m s} \mathscr{L}\left\{\frac{\partial^{2} \Psi_{0}(x, y, t)}{\partial x^{2}}+\frac{\partial^{2} \Psi_{0}(x, y, t)}{\partial y^{2}}\right\}\right\} \\
& -\mathscr{L}^{-1}\left\{\frac{i k}{2 s} \mathscr{L}\left\{\left(x^{2}+y^{2}\right) \Psi_{0}(x, y, t)\right\}\right\} \\
& -\mathscr{L}^{-1}\left\{\frac{i}{s} \mathscr{L}\left\{A_{0}\right\}\right\},
\end{aligned}
$$




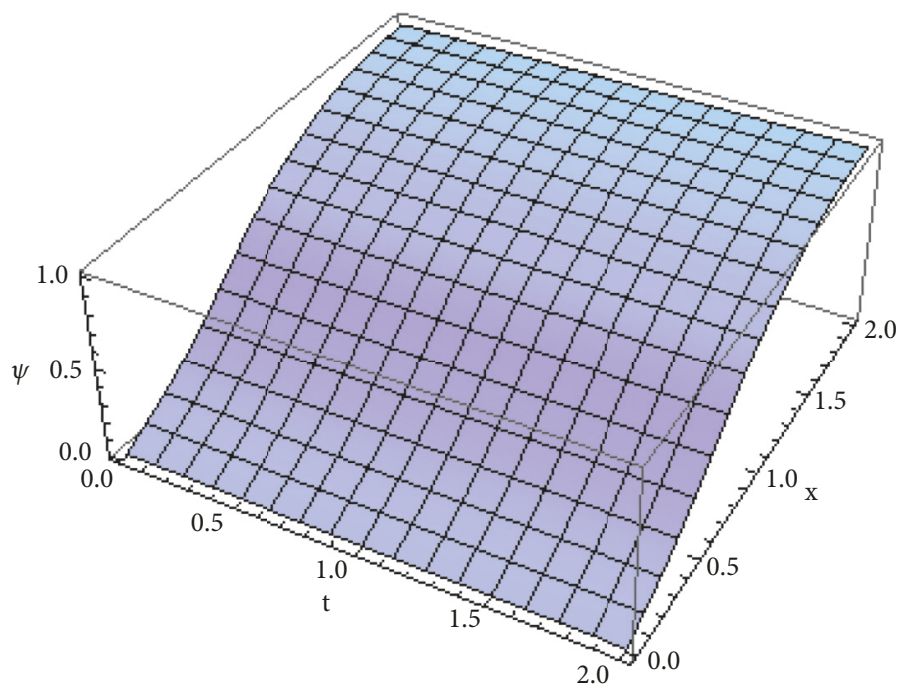

FIGURE 4: Imaginary solution of the one-dimensional wave function obtained by NDsolve.

$\Psi_{2}$

$$
\begin{aligned}
= & \mathscr{L}^{-1}\left\{\frac{i}{2 m s} \mathscr{L}\left\{\frac{\partial^{2} \Psi_{1}(x, y, t)}{\partial x^{2}}+\frac{\partial^{2} \Psi_{1}(x, y, t)}{\partial y^{2}}\right\}\right\} \\
& -\mathscr{L}^{-1}\left\{\frac{i k}{2 s} \mathscr{L}\left\{\left(x^{2}+y^{2}\right) \Psi_{1}(x, y, t)\right\}\right\} \\
& -\mathscr{L}^{-1}\left\{\frac{i}{s} \mathscr{L}\left\{A_{1}\right\}\right\},
\end{aligned}
$$

and thus

$$
\begin{aligned}
\Psi_{n} & =\mathscr{L}^{-1}\left\{\frac{i}{2 m s}\right. \\
& \left.\cdot \mathscr{L}\left\{\frac{\partial^{2} \Psi_{n-1}(x, y, t)}{\partial x^{2}}+\frac{\partial^{2} \Psi_{n-1}(x, y, t)}{\partial y^{2}}\right\}\right\} \\
& -\mathscr{L}^{-1}\left\{\frac{i k}{2 s} \mathscr{L}\left\{\left(x^{2}+y^{2}\right) \Psi_{n-1}(x, y, t)\right\}\right\} \\
& -\mathscr{L}^{-1}\left\{\frac{i}{s} \mathscr{L}\left\{A_{n-1}\right\}\right\} .
\end{aligned}
$$

Solving the above system of equations, we get

$$
\begin{aligned}
& \Psi_{0}(x, y, t)=e^{i(x+y)}, \\
& \Psi_{1}(x, y, t)=\frac{-i t}{m}\left(e^{i(x+y)}\right)-\frac{i k t e^{i(x+y)}}{2}\left(x^{2}+y^{2}\right) \\
& \quad-i t e^{i(x+y)}, \\
& \Psi_{2}(x, y, t)=\frac{-t^{2} e^{i(x+y)}}{(2 !) m^{2}}-\frac{k t^{2} e^{i(x+y)}}{(2 !) m}\left(x^{2}+y^{2}\right)
\end{aligned}
$$

$$
\begin{aligned}
& -\frac{2 t^{2} e^{i(x+y)}}{(2 !) m}+\frac{k t^{2} e^{i(x+y)}}{(2 !) m}+\frac{i k t^{2} e^{i(x+y)}(x+y)}{(2 !) m} \\
& -\frac{k^{2} e^{i(x+y)}\left(x^{2}+y^{2}\right)^{2} t^{2}}{4(2 !)}-\frac{t^{2} e^{i(x+y)}}{(2 !)} \\
& -\frac{k e^{i(x+y)} t^{2}\left(x^{2}+y^{2}\right)}{(2 !)},
\end{aligned}
$$$$
\Psi_{3}(x, y, t)=\frac{t^{3}}{3 !}\left(\frac{i e^{i(x+y)}}{m^{3}}+\frac{3 k(x+y) e^{i(x+y)}}{m^{2}}\right.
$$$$
-\frac{5 i k e^{i(x+y)}}{m^{2}}+\frac{3 k(x+y) e^{i(x+y)}}{m}
$$$$
+\frac{3 k^{2}\left(x^{2}+y^{2}\right)(x+y) e^{i(x+y)}}{2 m}
$$$$
+\frac{3 i k\left(x^{2}+y^{2}\right) e^{i(x+y)}}{2 m^{2}}-\frac{5 i k^{2}\left(x^{2}+y^{2}\right) e^{i(x+y)}}{2 m}
$$$$
+\frac{3 i k^{2}\left(x^{2}+y^{2}\right)^{2} e^{i(x+y)}}{4 m}+\frac{2 i e^{i(x+y)}}{m^{2}}-\frac{3 i k e^{i(x+y)}}{m}
$$$$
+\frac{3 i k\left(x^{2}+y^{2}\right) e^{i(x+y)}}{m}+\frac{3 i e^{i(x+y)}}{m}
$$$$
+\frac{i k^{3}\left(x^{2}+y^{2}\right)^{3} e^{i(x+y)}}{8}+\frac{3 i k\left(x^{2}+y^{2}\right) e^{i(x+y)}}{2}
$$$$
\left.+\frac{3 i k^{2}\left(x^{2}+y^{2}\right)^{2} e^{i(x+y)}}{4}+i e^{i(x+y)}\right) \text {. }
$$ 
Therefore, the approximate solution can be written as

$$
\begin{aligned}
\Psi & =\Psi_{0}+\Psi_{1}+\Psi_{2}+\Psi_{3}+\cdots \\
\Psi & =e^{i(x+y)}\left[1+\left(\frac{-i t}{m}-\frac{i k t}{2}\left(x^{2}+y^{2}\right)-i t\right)\right. \\
& +\frac{1}{2 !}\left(\frac{-i t}{m}-\frac{i k t}{2}\left(x^{2}+y^{2}\right)-i t\right)^{2} \\
& +\frac{t^{2}}{2 !}\left(\frac{i k(x+y)}{m}+\frac{k}{m}\right)+\frac{1}{3 !}\left(\frac{-i t}{m}\right. \\
& \left.-\frac{i k t}{2}\left(x^{2}+y^{2}\right)-i t\right)^{3}+\frac{t^{3}}{3 !}\left(\frac{3 k(x+y)}{m^{2}}-\frac{5 i k}{m^{2}}\right. \\
& +\frac{3 k(x+y)}{m}+\frac{3 k^{2}\left(x^{2}+y^{2}\right)(x+y)}{2 m} \\
& \left.\left.-\frac{5 i k^{2}\left(x^{2}+y^{2}\right)}{2 m}-\frac{3 i k}{m}\right)+\cdots\right] .
\end{aligned}
$$

5.2. HPM. In this section we apply the HPM to solve the two-dimensional equation; first we construct the following suitable homotopy:

$$
\begin{aligned}
& H(v, p)=(1-p)\left[\frac{\partial v}{\partial t}-\frac{\partial v_{0}}{\partial t}\right]+p\left[\frac{\partial v}{\partial t}\right. \\
& \left.\quad-\frac{i}{2 m}\left(\frac{\partial^{2} v}{\partial x^{2}}+\frac{\partial^{2} v}{\partial y^{2}}\right)+\frac{i k}{2}\left(x^{2}+y^{2}\right) v+i|v|^{2} v\right] \\
& \quad=0 .
\end{aligned}
$$

Using the same steps as in the one-dimensional case, we get the following system of equations:

$$
\begin{aligned}
& p^{0}: \frac{\partial v}{\partial t}-\frac{\partial v_{0}}{\partial t}=0, \\
& v_{0}(x, y, 0)=e^{i(x+y)}, \\
& p^{1}: \frac{\partial v_{1}}{\partial t}=\frac{i}{2 m}\left(\frac{\partial^{2} v_{0}}{\partial x^{2}}+\frac{\partial^{2} v_{0}}{\partial y^{2}}\right)-\frac{i k}{2}\left(x^{2}+y^{2}\right) v_{0} \\
& \quad-i\left|v_{0}\right|^{2} v_{0}, \\
& v_{1}(x, y, 0)=0, \\
& p^{j}: \frac{\partial v_{j}}{\partial t}=\frac{i}{2 m}\left[\frac{\partial^{2} v_{j-1}}{\partial x^{2}}+\frac{\partial^{2} v_{j-1}}{\partial y^{2}}\right] \\
& \quad-\frac{i k}{2}\left(x^{2}+y^{2}\right) v_{j-1}-i \sum_{i=0}^{i-1} \sum_{k=0}^{j-i-1}\left|v_{i}\right| v_{k}\left|v_{j-k-i-1}\right|, \\
& v_{j}(x, y, 0)=0,
\end{aligned}
$$

The general $j^{\text {th }}$ term can be obtained as follows:

$$
\begin{aligned}
v_{j} & =\int_{0}^{t}\left(\frac{i}{2 m}\left[\frac{\partial^{2} v_{j-1}}{\partial x^{2}}+\frac{\partial^{2} v_{j-1}}{\partial y^{2}}\right]-\frac{i k}{2}\left(x^{2}+y^{2}\right) v_{j-1}\right. \\
& \left.-i \sum_{i=0}^{i-1} \sum_{k=0}^{j-i-1}\left|v_{i}\right| v_{k}\left|v_{j-k-i-1}\right|\right) d \tau .
\end{aligned}
$$

Therefore, we can now evaluate the solution to the above system of differential equations:

$$
\begin{aligned}
& v_{0}(x, y, t)=e^{i(x+y)}, \\
& v_{1}(x, y, t)=\frac{-i t}{m}\left(e^{i(x+y)}\right)-\frac{i k t e^{i(x+y)}}{2}\left(x^{2}+y^{2}\right) \\
& -i t e^{i(x+y)} \text {, } \\
& v_{2}(x, y, t)=\frac{-t^{2} e^{i(x+y)}}{(2 !) m^{2}}-\frac{k t^{2} e^{i(x+y)}}{(2 !) m}\left(x^{2}+y^{2}\right) \\
& -\frac{2 t^{2} e^{i(x+y)}}{(2 !) m}+\frac{k t^{2} e^{i(x+y)}}{(2 !) m}+\frac{i k t^{2} e^{i(x+y)}(x+y)}{(2 !) m} \\
& -\frac{k^{2} e^{i(x+y)}\left(x^{2}+y^{2}\right)^{2} t^{2}}{4(2 !)}-\frac{t^{2} e^{i(x+y)}}{(2 !)} \\
& -\frac{k e^{i(x+y)} t^{2}\left(x^{2}+y^{2}\right)}{(2 !)} \\
& v_{3}(x, y, t)=\frac{t^{3}}{3 !}\left(\frac{i e^{i(x+y)}}{m^{3}}+\frac{3 k(x+y) e^{i(x+y)}}{m^{2}}\right. \\
& -\frac{5 i k e^{i(x+y)}}{m^{2}}+\frac{3 k(x+y) e^{i(x+y)}}{m} \\
& +\frac{3 k^{2}\left(x^{2}+y^{2}\right)(x+y) e^{i(x+y)}}{2 m} \\
& +\frac{3 i k\left(x^{2}+y^{2}\right) e^{i(x+y)}}{2 m^{2}}-\frac{5 i k^{2}\left(x^{2}+y^{2}\right) e^{i(x+y)}}{2 m} \\
& +\frac{3 i k^{2}\left(x^{2}+y^{2}\right)^{2} e^{i(x+y)}}{4 m}+\frac{2 i e^{i(x+y)}}{m^{2}}-\frac{3 i k e^{i(x+y)}}{m} \\
& +\frac{3 i k\left(x^{2}+y^{2}\right) e^{i(x+y)}}{m}+\frac{3 i e^{i(x+y)}}{m} \\
& +\frac{i k^{3}\left(x^{2}+y^{2}\right)^{3} e^{i(x+y)}}{8}+\frac{3 i k\left(x^{2}+y^{2}\right) e^{i(x+y)}}{2} \\
& \left.+\frac{3 i k^{2}\left(x^{2}+y^{2}\right)^{2} e^{i(x+y)}}{4}+i e^{i(x+y)}\right) \text {. }
\end{aligned}
$$

Hence, the approximate solution to (36), (37) is

$$
\begin{aligned}
\Psi & =e^{i(x+y)}\left[1+\left(\frac{-i t}{m}-\frac{i k t}{2}\left(x^{2}+y^{2}\right)-i t\right)\right. \\
& +\frac{1}{2 !}\left(\frac{-i t}{m}-\frac{i k t}{2}\left(x^{2}+y^{2}\right)-i t\right)^{2}
\end{aligned}
$$




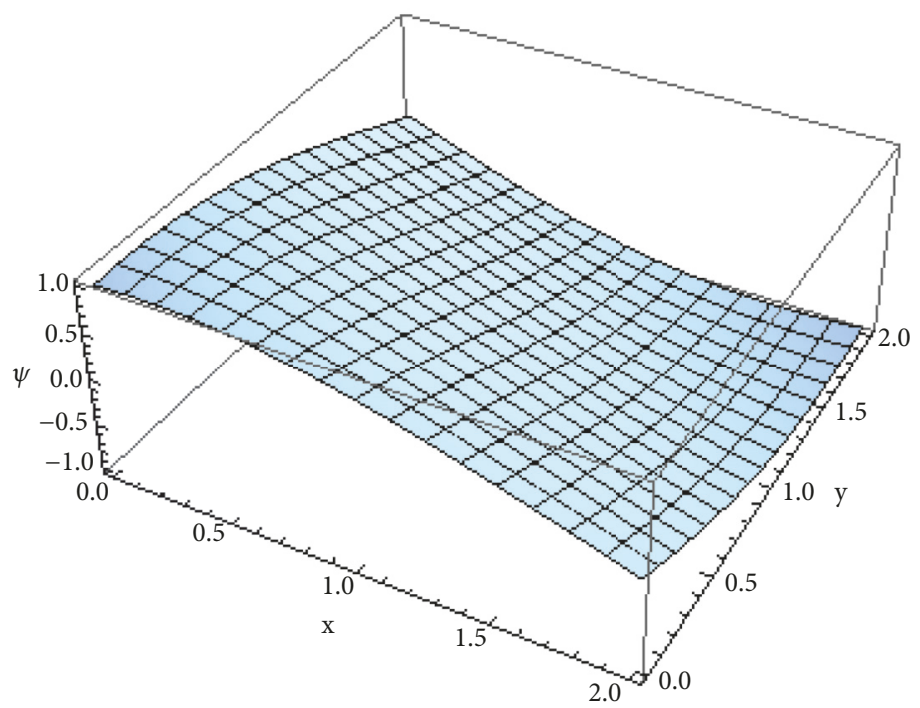

FIGURE 5: Real solution of the two-dimensional wave function obtained by LADM and HPM.

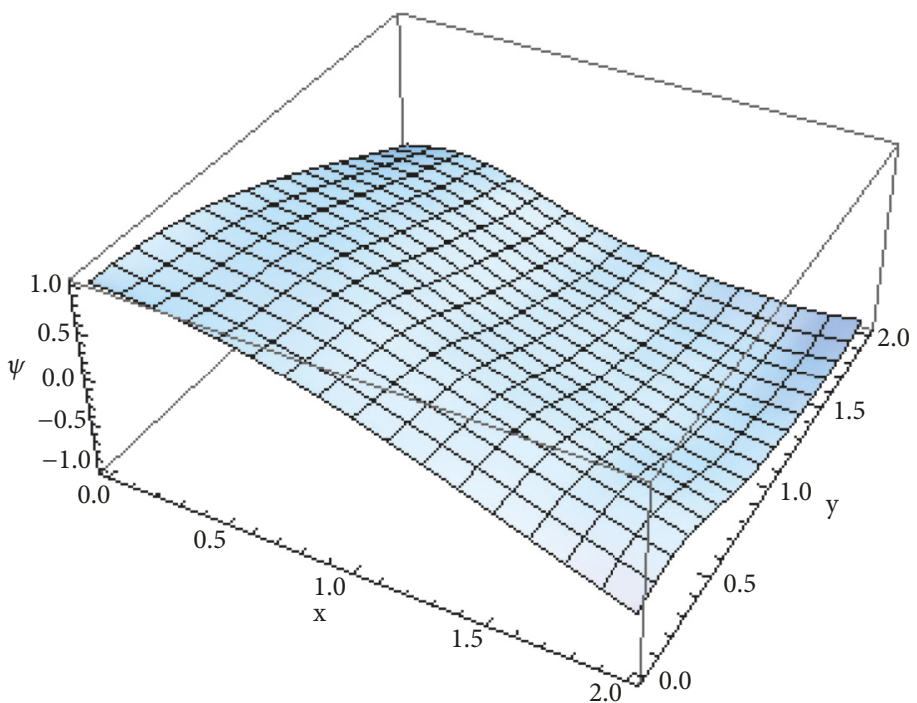

FIGURE 6: Real solution of the two-dimensional wave function obtained by NDsolve.

$+\frac{t^{2}}{2 !}\left(\frac{i k(x+y)}{m}+\frac{k}{m}\right)+\frac{1}{3 !}\left(\frac{-i t}{m}\right.$

$\left.-\frac{i k t}{2}\left(x^{2}+y^{2}\right)-\mathrm{it}\right)^{3}+\frac{t^{3}}{3 !}\left(\frac{3 k(x+y)}{m^{2}}-\frac{5 i k}{m^{2}}\right.$

$+\frac{3 k(x+y)}{m}+\frac{3 k^{2}\left(x^{2}+y^{2}\right)(x+y)}{2 m}$

$\left.\left.-\frac{5 i k^{2}\left(x^{2}+y^{2}\right)}{2 m}-\frac{3 i k}{m}\right)+\cdots\right]$.
The graphs of the solution of the two-dimensional wave function are shown in Figures 5, 6, 7, and 8. Figure 5 is the graph of the third-order approximation for the solution of the real part of the wave function obtained by LADM and HPM. Figure 6 is the graph of the wave function obtained using Mathematica function NDsolve. Figures 7 and 8 show the imaginary part of the solution. We use $k=m=1$ and $t=0.1$ in the calculations.

\section{Conclusion}

In this paper, homotopy perturbation and Laplace-Adomian decomposition methods have proven successful when used to 


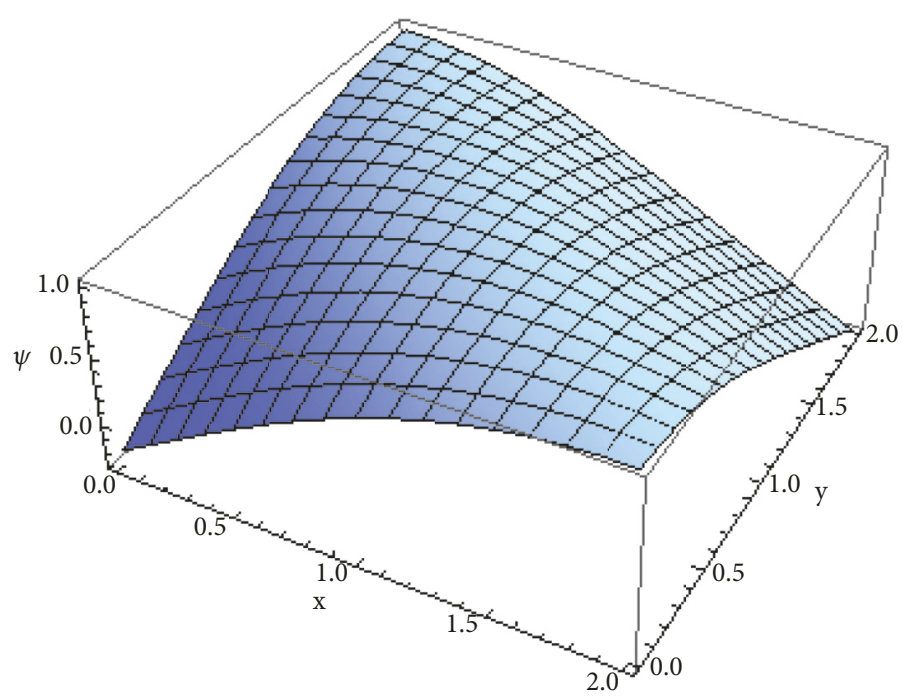

FIGURE 7: Imaginary solution of the two-dimensional wave function obtained by LADM and HPM.

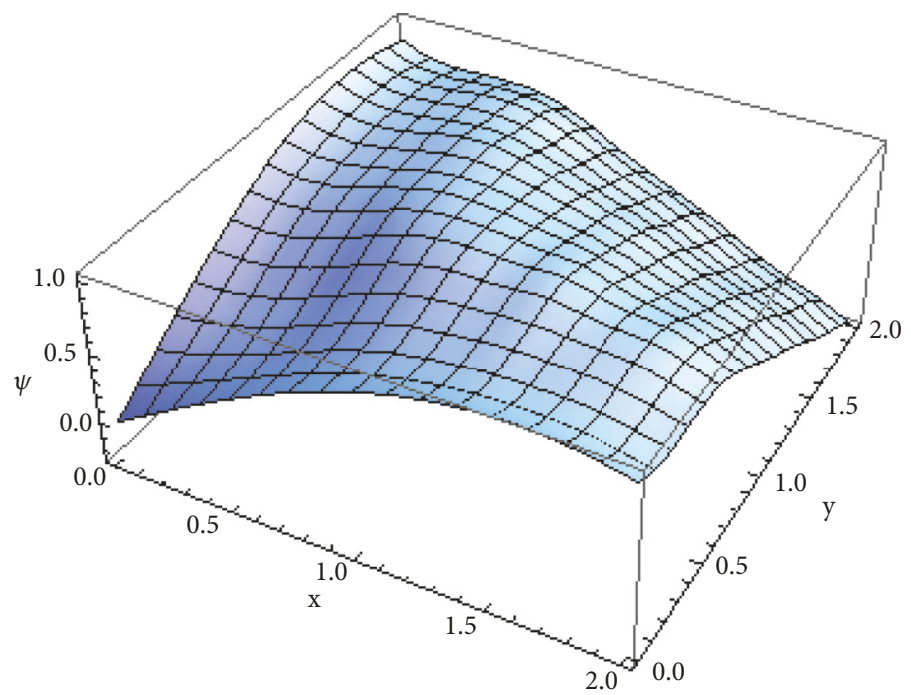

FIGURE 8: Imaginary solution of the two-dimensional wave function obtained by NDsolve.

find the approximate solution to the nonlinear Schrödinger equation with harmonic oscillator in one and in two dimensions. Our theoretical analyses have shown that both methods have given equivalent analytical approximate solutions successfully and efficiently. Comparison between HPM and LADM shows that although the results of these two methods when applied to solve the Schrödinger equation are in good agreement, HPM can overcome the difficulties arising in calculation of Adomian's polynomials. The solutions have been obtained and plotted for the real and imaginary wave function with the effect of adding the harmonic oscillator to the nonlinear Schrödinger equation in one and in two dimensions. HPM and LADM methods numerical results are in agreement with the solution obtained using Mathematica function NDsolve.

\section{Data Availability}

No data is involved in this research.

\section{Conflicts of Interest}

The authors declare no conflicts of interest.

\section{References}

[1] J. Cresser, Quantum Physics Notes, Department of Physics, Macquarie University, Australia, 2011, Quantum Physics Notes. Department of Physics, Macquarie University.

[2] D. J. Griffiths and D. F. Schroeter, Introduction to Quantum Mechanics, Cambridge University Press, 2018. 
[3] J. Biazar, R. Ansari, K. Hosseini, and P. Gholamin, "Solution of the linear and non-linear schrodinger equations using homotopy perturbation and Adomian decomposition methods," International Mathematical Forum. Journal for Theory and Applications, vol. 3, no. 37-40, pp. 1891-1897, 2008.

[4] A. Sadighi and D. D. Ganji, "Analytic treatment of linear and nonlinear schrodinger equations: a study with homotopyperturbation and Adomian decomposition methods," Physics Letters A, vol. 372, no. 4, pp. 465-469, 2008.

[5] P. Moradweysi, R. Ansari, K. Hosseini, and F. Sadeghi, "Application of modified Adomian decomposition method to pull-in instability of nano-switches using nonlocal Timoshenko beam theory," Applied Mathematical Modelling, vol. 54, pp. 594-604, 2018.

[6] J. Biazar and K. Hosseini, "An effective modification of Adomian decomposition method for solving Emden-Fowler type systems," National Academy of Science Letters, vol. 40, no. 4, pp. 285-290, 2017.

[7] J. Biazar and K. Hosseini, "A modified Adomian decomposition method for singular initial value Emden-Fowler type equations," International Journal of Applied Mathematics Research, vol. 5, no. 1, p. 69, 2016.

[8] J. Biazar and K. Hosseini, "Analytic approximation of Volterra's population model," Journal of Applied Mathematics \& Informatics, vol. 13, no. 1, pp. 5-17, 2017.

[9] R. I. Nuruddeen, "Elzaki decomposition method and its applications in solving linear and nonlinear Schrodinger equations," Sohag Journal of Mathematics, vol. 4, no. 2, pp. 1-5, 2017.

[10] A. Wazwaz, "A study on linear and nonlinear Schrodinger equations by the variational iteration method," Chaos, Solitons \& Fractals, vol. 37, no. 4, pp. 1136-1142, 2008.

[11] A. Niknam, A. A. Rajabi, and M. Solaimani, "Solutions of Ddimensional Schrodinger equation for Woods-Saxon potential with spin-orbit, coulomb and centrifugal terms through a new hybrid numerical fitting Nikiforov-Uvarov method," Journal of Theoretical and Applied Physics, vol. 10, no. 1, pp. 53-59, 2016.

[12] A. K. Alomari, M. S. Noorani, and R. Nazar, "Explicit series solutions of some linear and nonlinear Schrodinger equations via the homotopy analysis method," Communications in Nonlinear Science and Numerical Simulation, vol. 14, no. 4, pp. 11961207, 2009.

[13] S. Liao, Beyond perturbation: introduction to the homotopy analysis method, CRC press, 2003.

[14] J. Jezierski and W. a. Marzantowicz, Homotopy methods in topological fixed and periodic points theory, vol. 3, Springer Science \& Business Media, Dordrecht, Netherlands, 2006.

[15] J. H. He, "An elementary introduction to the homotopy perturbation method," Computers \& Mathematics with Applications, vol. 57, no. 3, pp. 410-412, 2009.

[16] J. Biazar and H. Ghazvini, "Exact solutions for non-linear Schrödinger equations by He's homotopy perturbation method," Physics Letters A, vol. 366, no. 1-2, pp. 79-84, 2007.

[17] A. Borhanifar and R. Abazari, "Numerical study of nonlinear Schrödinger and coupled Schrödinger equations by differential transformation method," Optics Communications, vol. 283, no. 10, pp. 2026-2031, 2010.

[18] A. Shidfar, A. Molabahrami, A. Babaei, and A. Yazdanian, "A study on the d-dimensional Schrödinger equation with a power-law nonlinearity," Chaos, Solitons \& Fractals, vol. 42, no. 4, pp. 2154-2158, 2009.
[19] A. Shidfar, A. Molabahrami, A. Babaei, and A. Yazdanian, "A series solution of the Cauchy problem for the generalized ddimensional Schrödinger equation with a power-law nonlinearity," Computers \& Mathematics with Applications, vol. 59, no. 4, pp. 1500-1508, 2010.

[20] L. Zheng, T. Wang, X. Zhang, and L. Ma, "The nonlinear Schrödinger harmonic oscillator problem with small odd or even disturbances," Applied Mathematics Letters, vol. 26, no. 4, pp. 463-468, 2013.

[21] G. Amador, K. Colon, N. Luna, G. Mercado, E. Pereira, and E. Suazo, "On solutions for linear and nonlinear Schrödinger equations with variable coefficients: A computational approach," Symmetry, vol. 8, no. 6, 2016.

[22] Y. Khan and Q. Wu, "Homotopy perturbation transform method for nonlinear equations using $\mathrm{He}$ s s polynomials," Computers \& Mathematics with Applications, vol. 61, no. 8, pp. 1963-1967, 2011.

[23] M. Kaplan, K. Hosseini, F. Samadani, and N. Raza, "Optical soliton solutions of the cubic-quintic non-linear Schrödinger's equation including an anti-cubic term," Journal of Modern Optics, vol. 65, no. 12, pp. 1431-1436, 2018.

[24] K. Hosseini, A. Zabihi, F. Samadani, and R. Ansari, "New explicit exact solutions of the unstable nonlinear Schrödinger's equation using the expa and hyperbolic function methods," Optical and Quantum Electronics, vol. 50, no. 2, 2018.

[25] K. Hosseini, J. Manafian, F. Samadani, M. Foroutan, M. Mirzazadeh, and Q. Zhou, "Resonant optical solitons with perturbation terms and fractional temporal evolution using improved $\tan (\phi(\eta) / 2)$-expansion method and exp function approach," Optik - International Journal for Light and Electron Optics, vol. 158, pp. 933-939, 2018.

[26] K. Hosseini, F. Samadani, D. Kumar, and M. Faridi, "New optical solitons of cubic-quartic nonlinear Schrödinger equation," Optik - International Journal for Light and Electron Optics, vol. 157, pp. 1101-1105, 2018.

[27] K. Hosseini, D. Kumar, M. Kaplan, and E. Y. Bejarbaneh, "New Exact Traveling Wave Solutions of the Unstable Nonlinear Schrödinger Equations," Communications in Theoretical Physics, vol. 68, no. 6, pp. 761-767, 2017.

[28] G. Adomian, "Solution of physical problems by decomposition," Computers \& Mathematics with Applications, vol. 27, no. 9-10, pp. 145-154, 1994.

[29] G. Adomian, "A review of the decomposition method in applied mathematics," Journal of Mathematical Analysis and Applications, vol. 135, no. 2, pp. 501-544, 1988.

[30] E. K. Jaradat, W. A. Amer, and D. Aloqali, "Using Laplace Decomposition Method to Solve Nonlinear Klein- Gordon Equation," UPB Scientific Bulletin, vol. 80, no. 2, Series D, 2018.

[31] S. A. Khuri, "A Laplace decomposition algorithm applied to a class of nonlinear differential equations," Journal of Applied Mathematics, vol. 1, no. 4, pp. 141-155, 2001.

[32] S. Liao, "Homotopy analysis method: A new analytic method for nonlinear problems," Applied Mathematics and MechanicsEnglish Edition, vol. 19, no. 10, pp. 957-962, 1998.

[33] J. He, "Homotopy perturbation technique," Computer Methods Applied Mechanics and Engineering, vol. 178, no. 3-4, pp. 257262, 1999. 


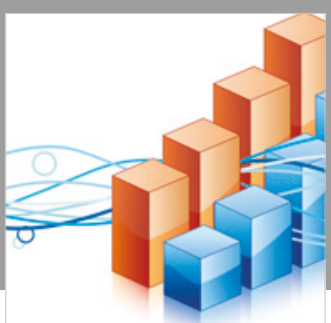

Advances in

Operations Research

\section{-n-m}
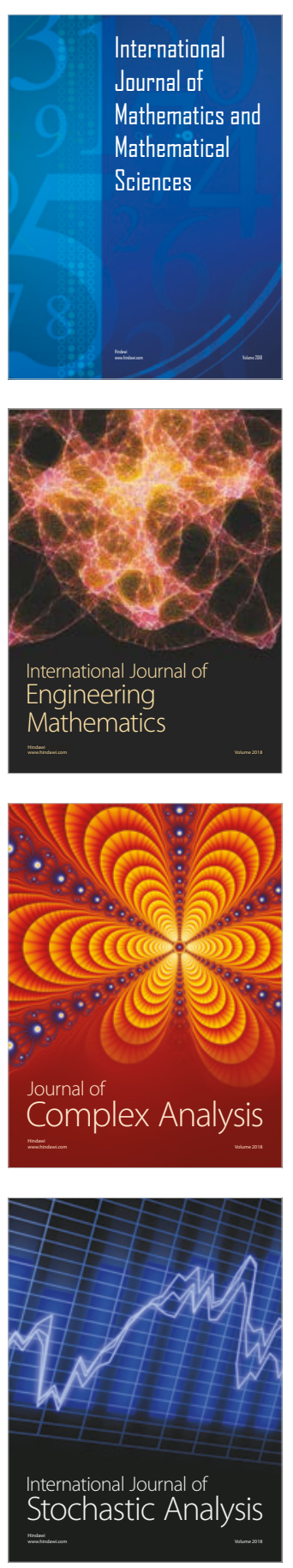
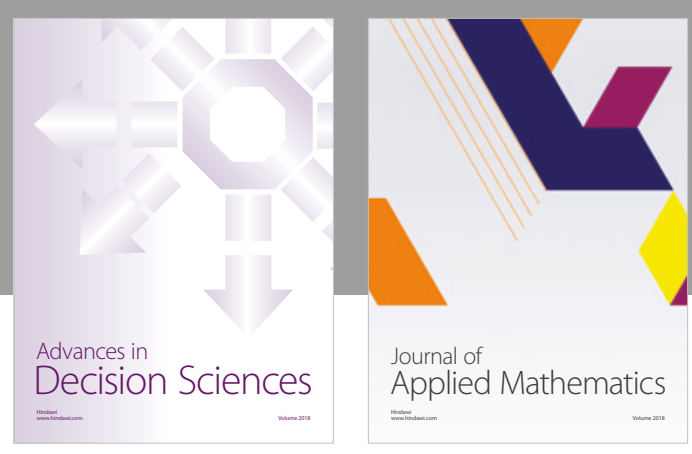

Journal of

Applied Mathematics
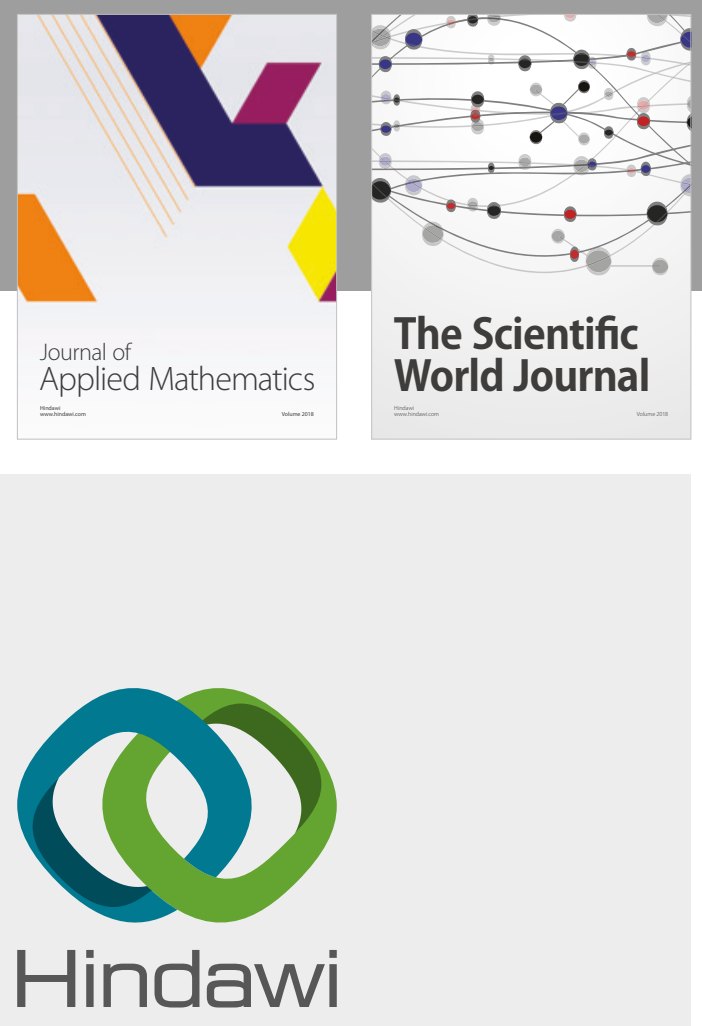

Submit your manuscripts at

www.hindawi.com

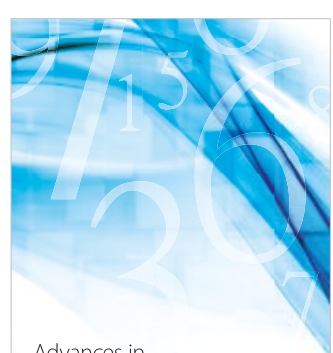

Advances in
Numerical Analysis
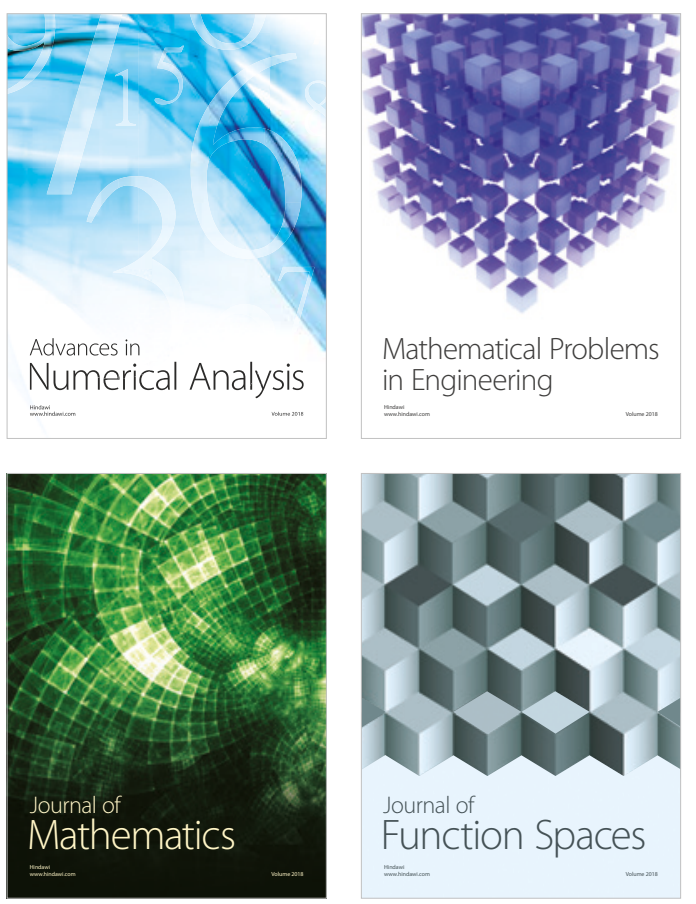

Mathematical Problems in Engineering

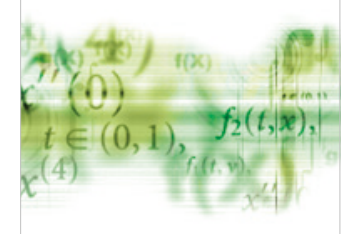

International Journal of

Differential Equations

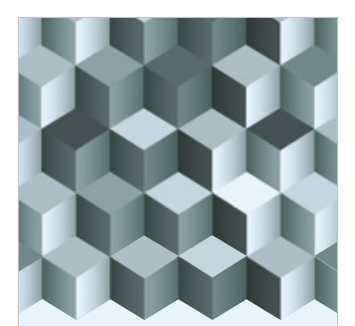

Journal of

Function Spaces

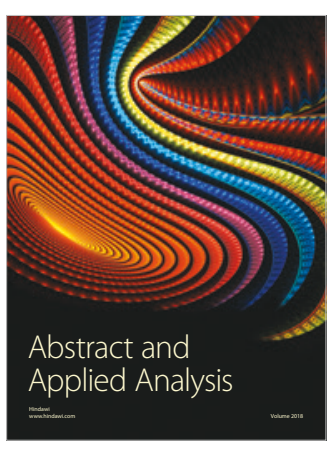

The Scientific

World Journal

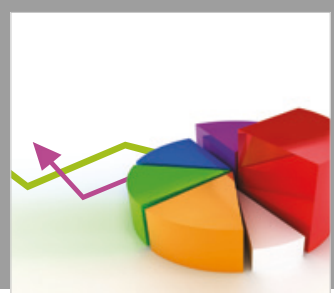

Journal of

Probability and Statistics
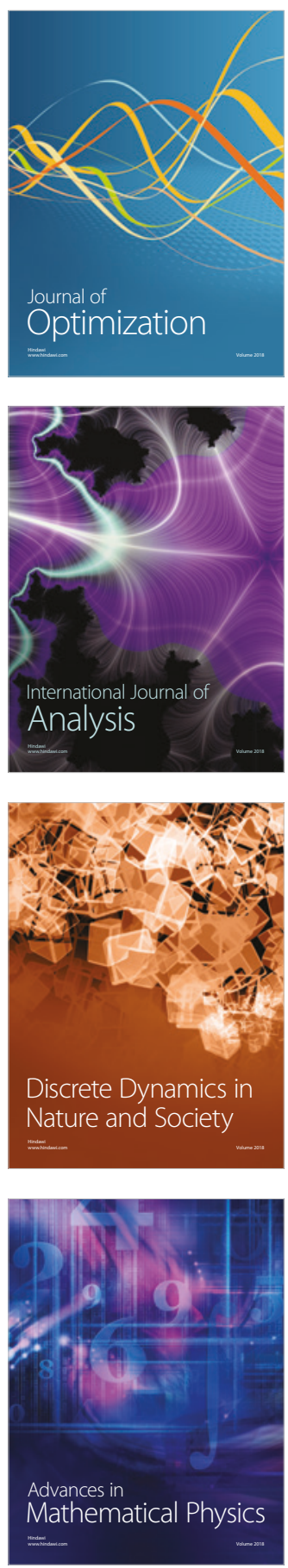